\title{
Investigation of chronic upper gastrointestinal haemorrhage
}

\author{
J.N. MacCaig \\ North Devon District Hospital, Barnstaple, Devon, UK.
}

\section{Introduction}

Acute haemorrhage from the upper alimentary tract is a common cause of emergency admission to hospital, and in about $80 \%$ of these patients the condition causing the bleeding will be an acute haemorrhagic gastritis or a chronic gastric or duodenal ulcer. The diagnosis in this group of patients will be confirmed by early endoscopic examination in about $90 \%$ of patients. A small proportion of these patients will go on to have recurrent episodes of haemorrhage over a period of months or years, leading to chronic blood loss and in many the diagnosis of the underlying cause of bleeding will be elusive. In addition, there are some lesions in the upper alimentary tract which will give rise to low grade chronic or occult bleeding and the presenting features will be those of chronic anaemia. Patients with chronic upper alimentary blood loss may be suffering from a very wide range of pathological conditions, some of them quite rare; the diagnostic challenge to the clinician may be great.

\section{Anaemia}

The majority of patients with chronic upper alimentary haemorrhage will present with iron deficiency anaemia rather than overt bleeding. The true nature of the anaemia is first established by the microcytic hypochromic blood picture and low serum ferritin and in case of doubtful information with these tests, marrow aspiration samples should be examined for depletion of the iron stores.

Identification of the gut as the source of blood loss may be difficult, but in the absence of a history of significant bleeding from some other organ system the gut should be assumed to be the site of blood loss in all adults except perhaps premenopausal women. The Haemoccult test which produces fewer false positive reactions (Ross \& Grey, 1964) than the Haemostix test, is probably the best available (Barrison et al.,

Correspondence: J.N. MacCaig, M.B., F.R.C.P.

Accepted: 8 November 1985
1981) but it fails to detect some colonic lesions which may be bleeding slowly or intermittently (Winawer, 1980). However, negative results from faecal occult blood testing can never exclude the possibility of intermittent blood loss and undue reliance on these tests can lead to a false sense of security about the absence of alimentary disease. Even this test gives positive results in elderly patients, only two thirds of whom can be shown to have a bleeding lesion (Bedford \& Wollner, 1958).

\section{Isotope methods of investigation}

When the patients' red cells are labelled with ${ }^{51} \mathrm{Cr}$ and reinjected, the faecal blood loss can be estimated by measurement of faecal radioactivity, and this is a more accurate and elegant method than the chemical methods of estimation for occult blood. An alternative approach is to give ${ }^{59} \mathrm{Fe}$ orally or intravenously, which in iron deficient patients is readily incorporated into new red cells. In the absence of blood loss from the body the level of the radioactivity of blood falls very slowly, and this measurement is most satisfactorily made by whole body counting (Callender et al., 1966). A fall in the body counts indicates blood loss, and this method can be very helpful in assessing blood loss in the inter-menstrual phase in premenstrual women with anaemia, or assessing the efficacy of hysterectomy in controlling blood loss, when both uterine and alimentary bleeding may coexist (Callender, 1981).

\section{Clinical evaluation}

Most chronic gastrointestinal disease can lead to either recurrent overt, or chronic occult haemorrhage (Korman, 1982). Hence clinical examination should start with a complete history; dyspeptic symptoms may suggest oesophagitis, gastritis, or a chronic peptic ulcer, while anorexia and weight loss are more indicative of carcinoma of stomach or pancreas, and 
dysphagia of carcinoma of the oesophagus. Intestinal colic and distension implying partial intestinal obstruction would suggest an intestinal tumour or stricture. A full history of both prescribed and self administered drug intake is vital, and aspirin is the most commonly incriminated drug as a cause of anaemia, being relevant in $8 \%$ in the Oxford Study (Callender, 1981). All the non-steroidal anti-inflammatory drugs have also been incriminated. A past history of abdominal surgery may provide a vital clue to the cause of anaemia, and of particular interest may be vascular surgery for grafting of abdominal arteries. Past history of residence abroad would indicate the need for stool examination for ova, cysts or segments of tapeworm, hookworm or other parasites.

Clinical examination should include a careful search for skin manifestation of haemorrhagic diseases such as hereditary haemorrhagic telangiectasia or pseudoxanthoma elasticum, or signs of purpura, and a history of familial haemorrhagic disorder should be sought. Mucocutaneous pigment of Peutz-Jeghers syndrome, stigmata of chronic liver disease, erythema nodosum suggesting associated inflammatory bowel disease, dermatitis herpetiformis and acanthosis nigrans, neurofibromatosis or skin angiomata may all be relevant. Abdominal examination may reveal an abnormal mass, or stigmata of chronic liver disease.

\section{Endoscopy}

Upper alimentary endoscopy should provide diagnostic information in the majority of these patients who have upper alimentary disease (Morrissey, 1977). This information may establish the diagnosis directly or indicate the source from which bleeding is coming, for example by showing blood arising from the papilla of Vater.

\section{Radiology}

Double contrast radiology is preferred to the single contrast method in the demonstration of upper alimentary disease, since the diagnostic yield of small and superficial mucosal lesions is higher, and the error rate lower at $6 \%$ for double contrast compared with $22 \%$ for single contrast techniques (Herlinger, 1981).

Angiography should be performed, particularly where endoscopy and barium meal examinations are negative (Classen et al., 1981). All appropriate arteries should be catheterized individually to include any possible lesion and the yield of information will be much greater if the lesion is actively bleeding at the time when the contrast medium is injected (Sheedy et al., 1975), in which case the material will be seen to escape into the gut lumen, provided that the rate of bleeding is $0.5 \mathrm{ml} / \mathrm{min}$ or more. Too aggressive an approach to arteriography may yield a high proportion of negative tests and a greater risk to patients (Keller \& Rosch, 1981).

Hiatal hernia alone is not a cause of gastrointestinal haemorrhage but associated complications such as oesophagitis, gastritis, or peptic ulceration of the oesophagus or stomach may bleed. The presence of a diverticulum in the upper alimentary tract is not usually accepted as a cause of bleeding, but such diverticula may ulcerate and give rise to bleeding (Gibbons \& Harvey, 1984). In such cases endoscopy and double contrast barium meal should be performed and the information combined.

Meckel's diverticula may bleed, and are difficult to demonstrate though both mesenteric angiography (Dalinka \& Wunder, 1973) and radioisotope imaging during an episode of bleeding may be diagnostic (Elsenberg \& Sherwood, 1975).

Haemobilia is a rare but important cause of chronic alimentary bleeding and over $50 \%$ of such patients will have suffered injury from blunt trauma or invasive investigations as the precipitating cause. Vascular malformations or tumours will explain $40 \%$ of the remaining cases.

\section{Arterio-enteric fistulae}

Spontaneous arterio-enteric fistulae may occur wherec the gut is tethered to the posterior abdominal wall at the fourth part of the duodenum, or into organs nearby such as the pancreas. After grafting of abdominal aneurysms any part of the gut may become tethered to the site of anastamosis and bleeding into the lumen arise at that point. Seven cases of bleeding from true aneurysms formed in the peripancreatic blood vessels, or pseudoaneurysms after vascular leakage into pancreatic pseudocysts are reported by Steckman et al. (1984), and selective angiography with abdominal ultrasound examination was helpful in diagnosis. In these patients a history of chronic relapsing pancreatitis, alcoholism or pancreatic pseudocyst was present, and treatment by embolotherapy beneficial. Other abdominal injury, particularly gun shot wounds may predispose to arterioenteric fistulae.

A further unusual case of bleeding from the pancreatic duct is reported in this journal (Ribbans et al., 1985) where arteriography showed a blocked dorsal pancreatic artery, and absent splenic vein, but did not define the source of haemorrhage. Ultrasound examination revealed a cystic mass in the pancreas subsequently shown at laparotomy to be an area of haemorrhage. Endoscopic retrograde cholangiopancreatography (ERCP) showed deformity of the pancreatic duct consistent with chronic pancreatitis. 
Treatment by distal pancreatectomy and splenectomy was successful in stopping bleeding over a 2 y follow up period though no single bleeding site was found in the resected specimen.

\section{Abdominal ultrasound and CT scanning}

The non-invasive nature of abdominal ultrasound examination ensures its popularity as a diagnostic technique and its place in the investigation in the above groups of patients is clear. Diagnostic information of pathology in the liver, pancreas, abdominal aorta, and all major vessels will often be obtained. When combined with other techniques such as arteriography or ERCP the accuracy of diagnostic information is enhanced. Abdominal computed tomographic (CT) scanning will yield a similar quality of information and again be valuable in combination with the other techniques.

\section{Endoscopic retrograde cholangiopancreatography}

When the common causes of gastrointestinal bleeding have been excluded, and particularly if bleeding from the ampulla of Vater has been identified or suspected at routine endoscopy, then ERCP is indicated. This provides the best prospect of outlining the biliary tree and anatomy of the pancreatic ducts and is more likely to show pathology of the pancreas when there is a past history of alcoholism, chronic pancreatitis or even an acute attack of pancreatitis in the past.

\section{Small bowel enema and meal}

Examination of the small bowel by administration by mouth of dilute barium is a reliable, simple and acceptable technique for routine investigation (Lintott, 1978). Intubation of the small bowel is preferred by Herlinger (1981) because of more consistent results in demonstrating fine detail, particularly when a double contrast technique is used. Bleeding lesions such as Meckel's diverticulum, intussusception, Crohn's disease, and intramural bleeding may be demonstrated, and either benign or malignant tumours are rarely shown. Lymphomatous infiltration of the small bowel is even less common, and elsewhere in this journal a case of haemorrhage arising from small bowel capillary dilation is reported in a patient suffering from portal hypertension (Gallagher et al., 1985). This patient had cirrhosis and portal hyperten- sion, with persistent gastrointestinal blood loss and although she had small oesophageal varices there was no evidence that the bleeding had arisen from them. Biopsy of hyperaemic areas of duodenal mucosa showed grossly dilated capillaries in the villi, a finding previously reported by Astaldi \& Strosseli in 1960 . The hypothesis that the capillary dilatation may be the source of haemorrhage in some patients with portal hypertension merits further study.

\section{Laparotomy}

Despite the most extensive investigations a few patients may continue to have recurrent haemorrhage and anaemia, and it may prove impossible to define the precise source of bleeding. Such patients may have several potential pathological lesions which could bleed without it being possible to incriminate any one site at any one time as the origin of blood loss. Patients may have multiple angiomata or areas of angiodysplasia, the lesions in hereditary haemorrhagic telangiectasia are usually multiple, and small tumours in the small intestine may escape detection and yet bleed recurrently. Patients in these categories may require laparotomy to identify the bleeding site and enable a further surgical procedure to arrest the haemorrhage. At such a laparotomy it is important to be prepared to endoscope the lumen of the whole gut and the place of laparotomy in this situation is described by Williams (1981).

\section{Conclusion}

The majority of patients with chronic upper alimentary bleeding will have either a clear and easily defined pathology in the upper gastrointestinal tract and will require nothing more elaborate than a full clinical evaluation, upper gastrointestinal endoscopy and perhaps double contrast barium meal to establish a diagnosis. A minority of these patients will require a wide range of skilled investigation techniques ending up with exploratory laparotomy and perioperative intestinal endoscopy before the cause is found. In some patients no one investigation will solve the diagnostic problem, but combined evidence of ultrasound, arteriography, and ERCP may give the answer. In this clinical situation it is correct to pursue all available investigation techniques in order to establish an accurate diagnosis on which to plan treatment. 


\section{References}

ASTALDI, G. \& STROSSELI, E. (1960). Peroral biopsy of the intestinal mucosa in hepatic cirrhosis. American Journal of Digestive Diseases, 5, 603.

BARRISON, I.G., PRIMAVESI, J., GILMORE, I.T., LITTLEWOOD, E.R., SHARPLES, A. \& PARKIN, R.A. (1981). Screening for occult gastrointestinal bleeding in hospital patients. Journal of the Royal Society of Medicine, 74, 41.

BEDFORD, P.D. \& WOLLNER, L. (1958). Occult intestinal bleeding as a cause of anaemia in elderly people. Lancet, $\mathbf{i}$, 1145.

CALLENDER, S.T. (1981). Chronic blood loss. In Gastrointestinal Haemorrhage, p. 115. Wright P.S.G.: Bristol.

CALLENDER, S.T., WITTS, L.J. \& WARNER, G.T. (1966). The use of a simple whole body counter for haematological investigations. British Journal of Haematology, 12, 276.

ClASSEN, M., PHILliP, J. \& SMITH-LAING, G. (1981). Management of upper gastrointestinal haemorrhage. In Gastroenterology, 1, Foregut. Baron, J.H., F.G. (eds.). Butterworths: London.

DALINKA, M.K. \& WUNDER, J.F. (1973). Meckels diverticulum and its complications with emphasis on roentgenologic demonstration. Radiology, 106, 295.

ELSENBERG, D. \& SHERWOOD, C.E. (1975). Bleeding Meckel's diverticulum diagnosed by arteriography and radioisotope imaging. American Journal of Digestive Diseases, $20,573$.

GALLAGHER, C., BONAR, F., DEMPSEY, J. \& CROWE, J. (1985). Small bowel capillary dilatation in portal hypertension. Postgraduate Medical Journal, 61, 541.

GIBBONS, C.P. \& HARVEY, L. (1984). An ulcerated gastric diverticulum - a rare cause of haematemesis and melaena. Postgraduate Medical Journal, 60, 693.
HERLINGER, H. (1981). Consensus development conference on endoscopy. Digestive Diseases and Sciences, 26, Suppl. $76 \mathrm{~S}$.

KELLER, F.S. \& ROSCH, J. (1981). Value of angiography in diagnosis and therapy of acute upper gastrointestinal haemorrhage. Digestive Diseases \& Sciences, 26, Suppl. $78 \mathrm{~S}$.

KORMAN, M.G. (1982). Acute upper gastrointestinal haemorrhage. Medicine (International), 13, 597.

LINTOTT, D.J. (1978). The small bowel meal. British Journal of Radiology, 51, 146.

MORRISSEY, J.F. (1977). Early endoscopy for major gastrointestinal bleeding - it should be done. American Journal of Digestive Diseases, 22, 534.

RIBBANS, W.J., POUNDER, R.E. \& LINDSAY, I. (1985) Haemorrhage through the ampulla of Vater. Postgraduate Medical Journal, 61, 533.

ROSS, G. \& GREY, C.H. (1964). Assessment of routine tests for occult blood in faeces. British Medical Journal, 1, 1351.

SHEEDY, P.F., FULTON, R.E. \& ATTWELL, D.T. (1975). Angiographic evaluation of patients with chronic gastrointestinal bleeding. American Journal of Roentgenology Radium Therapy and Nuclear Medicine, 123, 338.

STECKMAN, M.L., DOOLEY, M.C., JAQUES, P.F. \& POWELL, D.W. (1984). Major gastrointestinal haemorrhage from pancreatic blood vessels in pancreatitis. Treatment by embolotherapy. Digestive Diseases and Sciences, 29, 486.

WILLIAMS, J.A. (1981). Surgical management of acute intestinal bleeding. In Gastrointestinal Haemorrhage p. 360. Wright P.S.G.: Bristol.

WINAWER, S.J. (1980). Screening for colorectal cancer: an overview. Cancer, 45 (5 Suppl), 1093. 